\title{
THE INFLUENCE OF THE CORE ORTHOTROPY ON THE WRINKLING OF SANDWICH PANELS
}

\author{
Jolanta Pozorska ${ }^{1}$, Zbigniew Pozorski ${ }^{2}$ \\ ${ }^{I}$ Institute of Mathematics, Czestochowa University of Technology, Częstochowa, Poland \\ ${ }^{2}$ Institute of Structural Engineering, Poznan University of Technology, Poznań, Poland \\ jolanta.pozorska@im.pcz.pl,zbigniew.pozorski@put.poznan.pl
}

\begin{abstract}
The paper concerns the phenomenon of wrinkling of compressed facing of a sandwich panel. The parametric analysis of the numerical model of the sandwich panel with a soft core is presented. The core was assumed as an elastic orthotropic material. The results of numerical simulations show the sensitivity of a sandwich structure to the variations of the core parameters. The results are evaluated qualitatively and quantitatively.
\end{abstract}

Keywords: sandwich panels, wrinkling, soft core, orthotropic material

\section{Introduction}

In this paper panels composed of thin, smooth and rigid facings and a thick and soft core are examined. This type of panel is widely used as an envelope of buildings. Thanks to the thick core, sandwich panels are characterized by excellent thermal insulation and high load capacity with low weight. On the other hand, the heterogeneity of the structure of the panel results in the occurrence of different failure mechanisms. One of the most important forms of damage is face wrinkling during bending.

The problem of wrinkling was discussed in many scientific papers. The most important issue is to determine the load (or stress), which causes a local loss of stability (wrinkling) of the panel. In terms of the approach to the problem analytical methods [1-3], and experimental and numerical [4-6] can be distinguished. In most of the work, it is assumed that all layers of panels are made from homogeneous and isotropic materials. In fact, it turns out that some core materials are anisotropic. Somewhat to our surprise, we find that this also applies to polyurethane foams. Anisotropy is the result of the sandwich panel manufacturing process because, during production, growing foam cells are subjected to different interactions.

The problem of the core anisotropy was rarely discussed in the literature. The paper [7] is one of the first works taking this problem analytically. A similar approach was presented in [8], where analytical results were compared with the numerical model. The core anisotropy certainly is of particular importance in the case 
of non-uniform boundary conditions [9]. It is worth noting that the determination of the anisotropic parameters of the core is a very difficult issue. In [10], the mixed experimental numerical method of determination of material parameters of orthotropic foam was presented.

The aim of this study was to analyze the influence of the orthotropic core parameters on the wrinkling stress of the sandwich panel in bending. The problem is examined numerically. The model sensitivity with respect to the variation of the core parameters will indicate the parameters that are critical for behavior (and failure) of the sandwich panel.

\section{Description of the system}

To analyze the influence of the core orthotropy on the local loss of stability, the static system presented in Figure 1 was considered.

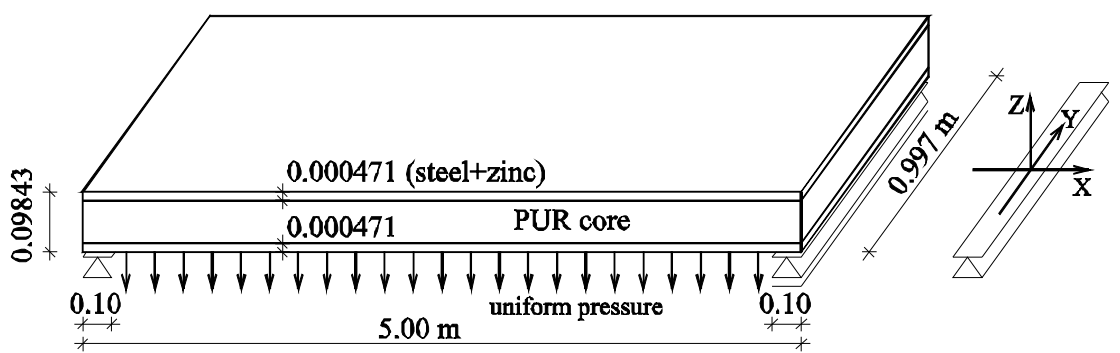

Fig. 1. The static system of a one-span sandwich panel

The sandwich panel with a length $5.00 \mathrm{~m}$ is located on two supports with a width $0.10 \mathrm{~m}$. The supports have the freedom of rotation around the $y$-axis. One of the supports is free to shift along the $x$-axis. The structure is loaded by uniform pressure perpendicular to the lower face of the sandwich. The loading is directed downwards, i.e. opposite to the $z$-axis, and causes tension of the lower face and compression of the upper face. The total depth of the panel is $98.43 \mathrm{~mm}$. The thickness of each of the faces is $t_{F}=0.471 \mathrm{~mm}$ and the depth of the core is $97.488 \mathrm{~mm}$. The width of the panel $b=0.997 \mathrm{~m}$.

The material parameters of the steel faces are: Young's modulus $E_{F}=195 \mathrm{GPa}$ and Poisson's ratio $v_{F}=0.3$. Moreover, the actual relationship between stress and strain was introduced. In the tensile test the yield strength was $360 \mathrm{MPa}$, and the tensile strength reached $436 \mathrm{MPa}$. The core is made of polyurethane foam. It was assumed that the foam is an orthotropic, linear elastic material. The linear elasticity was defined by 'engineering constants': the three moduli $E_{1}, E_{2}, E_{3}$, Poisson's ratios $v_{12}, v_{13}, v_{23}$ and the shear moduli $G_{12}, G_{13}$ and $G_{23}$, associated with the material's principal directions $\mathrm{x}_{1}, \mathrm{x}_{2}, \mathrm{x}_{3}$. These 9 parameters define the relation between strain and stress components (engineering notation was used): 


$$
\left\{\begin{array}{l}
\varepsilon_{11} \\
\varepsilon_{22} \\
\varepsilon_{33} \\
\gamma_{12} \\
\gamma_{13} \\
\gamma_{23}
\end{array}\right\}=\left[\begin{array}{cccccc}
1 / E_{1} & -v_{21} / E_{2} & -v_{31} / E_{3} & 0 & 0 & 0 \\
-v_{12} / E_{1} & 1 / E_{2} & -v_{32} / E_{3} & 0 & 0 & 0 \\
-v_{13} / E_{1} & -v_{23} / E_{2} & 1 / E_{3} & 0 & 0 & 0 \\
0 & 0 & 0 & 1 / G_{12} & 0 & 0 \\
0 & 0 & 0 & 0 & 1 / G_{13} & 0 \\
0 & 0 & 0 & 0 & 0 & 1 / G_{23}
\end{array}\right]\left\{\begin{array}{c}
\sigma_{11} \\
\sigma_{22} \\
\sigma_{33} \\
\sigma_{12} \\
\sigma_{13} \\
\sigma_{23}
\end{array}\right\} .
$$

In general, $v_{12} \neq v_{21}$, but the ratios are related by $v_{\mathrm{ij}} / E_{\mathrm{i}}=v_{\mathrm{ji}} / E_{\mathrm{j}}$. Due to the nature of the production process, it was assumed that the material's principal directions $x_{1}$, $x_{2}, x_{3}$ correspond to the axes of system of coordinates $x, y, z$ (cf. Fig. 1). Of course, the parameter values must fulfill the relevant conditions for material stability.

The numerical model of the system was developed in [11]. Facings were modeled using four node, doubly curved, thin or thick shell, reduced integration, hourglass control and finite membrane strain elements S4R. The core of the panel was modeled using eight node linear brick elements C3D8R. The geometrically nonlinear static analysis of the problem was used. The Riks' method was applied. For the brevity of interpretation of the results, interface layers were not implemented. Interaction between all parts was assumed as TIE type, which makes equal displacements of nodes. Geometric imperfections were introduced as a combination of four buckling modes with the multiplier 0.005 .

\section{Parametric analysis}

The point of reference for all subsequent analyses is the isotropic core material: $E_{1}=E_{2}=E_{3}=8610 \mathrm{kPa}, v_{12}=v_{13}=v_{23}=0.02$ and $G_{12}=G_{13}=G_{23}=4220.6 \mathrm{kPa}$. Of course, the shear modules result from the basic relationship $G=E / 2(1+v)$. The name of the model with the isotropic core is si 21. All other models have an orthotropic core. In the model si_22, the following Poisson's ratios were changed: $v_{13}=0.2$ and $v_{23}=0.2$. It means that the ratios increased 10 times. In the model $s i$ 23 the parameters are the same as in the case of $s i 21$, with the only difference being that $v_{12}=0.2$. In the models si_24 and si_25 was set $E_{1}=E_{2}=11030 \mathrm{kPa}$ and $E_{1}=E_{2}=13450 \mathrm{kPa}$, respectively. The modulus in the direction perpendicular to the facings has been reduced in the model $s i 26\left(E_{3}=6130 \mathrm{kPa}\right)$ and $s i 27$ $\left(E_{3}=3650 \mathrm{kPa}\right)$. In models from si_21 to $s i \_27$, shear modules were equal to $4220.6 \mathrm{kPa}$. The variation of the parameter $\bar{G}_{13}$ was introduced in $s i 28\left(G_{13}=\right.$ $=3600 \mathrm{kPa})$ and si $29\left(G_{13}=3000 \mathrm{kPa}\right)$.

The static analysis was performed for each model. In any case, the panel was damaged by wrinkling of the upper facing (compressed in bending). The wrinkling of the upper face in the model si_26 is presented in Figure 2. Because the two facings are of equal thickness, maximum tensile stress in the lower facing, corresponding 
to the maximum load, was adopted as the wrinkling stress. Of course, the wrinkling stress can not be read from the upper face because of large variation of the stress values. The results of wrinkling stress $\sigma_{w r}$ are presented in the column 3 of Table 1 . In the column 2 are described the changes of the core parameters in relation to the isotropic model $s i$ 21.

Table 1

The dependence of the wrinkling stress on the parameters of the core material

\begin{tabular}{|c|c|c|}
\hline $\begin{array}{c}\text { Name of the } \\
\text { model }\end{array}$ & $\begin{array}{c}\text { Changes with respect } \\
\text { to the reference model }\end{array}$ & $\begin{array}{c}\sigma_{w r} \\
{[\mathrm{MPa}]}\end{array}$ \\
\hline 1 & 2 & 3 \\
\hline si_21 & $\begin{array}{c}\text { The reference model } \\
\text { (the isotropic core material) }\end{array}$ & 158.4 \\
\hline si_22 & $v_{13}=v_{23}=0.2$ & 156.6 \\
\hline si_23 & $v_{12}=0.2$ & 158.5 \\
\hline si_24 & $E_{1}=E_{2}=11030 \mathrm{kPa}$ & 159.5 \\
\hline si_25 & $E_{1}=E_{2}=13450 \mathrm{kPa}$ & 160.4 \\
\hline si_26 & $E_{3}=6130 \mathrm{kPa}$ & 141.0 \\
\hline si_27 & $E_{3}=3650 \mathrm{kPa}$ & 117.7 \\
\hline si_28 & $G_{13}=3600 \mathrm{kPa}$ & 151.7 \\
\hline si_29 & $G_{13}=3000 \mathrm{kPa}$ & 144.2 \\
\hline
\end{tabular}

Analyzing the results shown in Table 1, it can be concluded that the Poisson's ratios have minimal impact on the value of wrinkling stress. Ten times higher ratios cause a change in wrinkling stress of $1 \%$. Similarly, in the case of changing modules $E_{1}$ and $E_{2}$. A noticeable change of these modules results in minimal effect. There can be nothing surprising because the panel has a high tensile stiffness in the direction of $x$ and $y$ resulting from the facings rigidity. Thus, even a large change in the core rigidity in these directions may not be significant, since the core stiffness is approximately 200 times smaller than the stiffness of the facings.

The most interesting case concerns a change of the modulus $E_{3}$. The decrease in $E_{3}$ (modulus in the direction perpendicular to the facings) causes much faster local instability of the compressed facing. The ratio of the two numerical values of wrinkling stress is surprisingly compatible with a relationship resulting from the application of theoretical equation for wrinkling stress for homogeneous materials:

$$
\sigma_{w r}=\mu \sqrt[3]{E_{F} E_{C} G_{C}}
$$

where $\mu$ is numerical coefficient, and $E_{C}, G_{C}$ are the Young's modulus and the shear modulus of the core material. Comparing models si_26 and si_27 to si_21, we get: 


$$
\begin{aligned}
& \frac{141.0}{158.4}=0.890 \approx \sqrt[3]{\frac{6130}{8610}}=0.893 \\
& \frac{117.7}{158.4}=0.743 \approx \sqrt[3]{\frac{3650}{8610}}=0.751 .
\end{aligned}
$$

The dependence of the wrinkling stress from the shear modulus $G_{13}$ looks very similar. It should first be noted that for the present panel, shear occurs mainly in the plane $\mathrm{x}_{1}-\mathrm{x}_{3}$. Reducing the $G_{13}$ modulus increases the displacements and deformations of the entire panel, so it obviously accelerates face wrinkling. It should come back to the relationship between wrinkling stress. Comparing models si_28 and si_29 to si_21, we obtain:

$$
\begin{aligned}
& \frac{151.7}{158.4}=0.957 \approx \sqrt[3]{\frac{3600}{4220.6}}=0.948 \\
& \frac{144.2}{158.4}=0.910 \approx \sqrt[3]{\frac{3000}{4220.6}}=0.892 .
\end{aligned}
$$

Of course, this consistency can not be perfect because in the core of the panel there are also shear stresses and deformations in other planes.

Let us return to the models $s i \_26$ and $s i$ 27. Decreasing $E_{3}$ reduces the wrinkling stress (as in si_28 and si_29), but it is understood that the change of $E_{3}$ does not affect the global displacement of the panel. It is therefore a different situation than in the case of models si_28 and si_29, wherein the reduction of $G_{13}$ increases the vertical displacements of the panel.

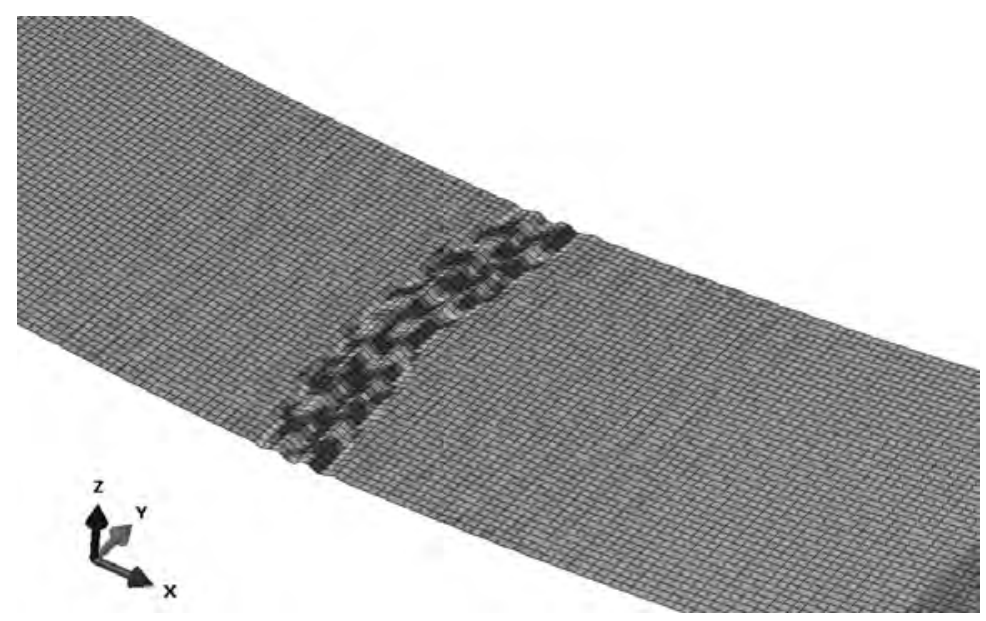

Fig. 2. Wrinkling of the upper face in the model si_26 


\section{Conclusions}

The influence of orthotropic core parameters on the local loss of stability of panels (or rather its facings) was investigated. The results showed that only two parameters of the core have a significant impact on the wrinkling stress: the modulus of elasticity $E_{3}$ (the direction perpendicular to the faces) and the shear modulus $G_{13}$ (corresponding to the classical shear of the core). Other core parameters have a marginal impact on the behavior of the panel. It should be noted that in contrast to $G_{13}$, the change of $E_{3}$ does not affect the global displacement of the sandwich panel. With some surprise it should also be noted that the change of wrinkling stress caused by changes of the key parameters of the orthotropic core, can be estimated using the known equation for the wrinkling stress used in the case of the isotropic core. Our observations concerning the sensitivity of sandwich panels induce even greater efforts to proper determination of the parameters of the core materials.

\section{References}

[1] Allen H.G., Analysis and Design of Structural Sandwich Panels, Pergamon Press Ltd., Oxford 1969.

[2] Plantema F.J., Sandwich Construction, John Wiley \& Sons, Inc., 1966.

[3] Hoff N.J., Mautner S.E., Bending and buckling of sandwich beams, Journal of Aeronautical Sciences 1948, 15(12), 707-720.

[4] Gdoutos E.E., Daniel I.M., Wang K.-A., Compression facing wrinkling of composite sandwich structures, Mechanics of Materials 2003, 35(3-6), 511-522.

[5] Stiftinger M.A., Rammerstorfer F.G., Face layer wrinkling in sandwich shells - theoretical and experimental investigations, Thin-walled Structures 1997, 29(1-4), 113-127.

[6] Koissin V., Shipsha A., Skvortsov V., Effect of physical nonlinearity on local buckling in sandwich beams, Journal of Sandwich Structures and Materials 2010, 12, 477-494.

[7] Norris C.B., Ericksen W.S., March H.W., Smith C.B., Boller K.H, Wrinkling of the facings of sandwich construction subjected to edgewise compression, Forest Products Laboratory Report 1810, 1949.

[8] Vonach W.K., Rammerstorfer F.G., Wrinkling of thick orthotropic sandwich plates under general loading conditions, Achive of Applied Mechanics 2000, 70, 338-348.

[9] Studziński R., Pozorski Z., Garstecki A., Structural behavior of sandwich panels with asymmetrical boundary conditions, Journal of Constructional Steel Research 2015, 104, 227-234.

[10] Chuda-Kowalska M., Gajewski T., Garbowski T., Mechanical characterization of orthotropic elastic parameters of a foam by the mixed experimental-numerical analysis, Journal of Theoretical and Applied Mechanics 2015, 53(2), 383-394.

[11] Pozorska J., Pozorski Z., The numerical model of sandwich panels used for specifying wrinkling stress, Journal of Applied Mathematics and Computational Mechanics 2014, 13(3), 187-194. 